\title{
Intrasession and Intersession Reliability of Running Mechanics During Treadmill Sprints
}

\author{
Olivier Girard, Franck Brocherie, Jean-Benoit Morin, and Grégoire P. Millet
}

\begin{abstract}
Purpose: To determine the intrasession and intersession (ie, within- and between-days) reliability in treadmill sprinting-performance outcomes and associated running mechanics. Methods: After familiarization, 13 male recreational sportsmen (team- and racket-sport background) performed three 5-s sprints on an instrumented treadmill with 2 min recovery on 3 different days, 5-7 $\mathrm{d}$ apart. Intrasession (comparison of the 3 sprints of the first session) and intersession (comparison of the average of the 3 sprints across days) reliability of performance, kinetics, kinematics, and spring-mass variables were assessed by intraclass correlation coefficient (ICC) and coefficients of variation (CV\%). Results: Intrasession reliability was high (ICC > .94 and CV < 8\%). Intersession reliability was good for performance indices $(.83<\mathrm{ICC}<.89$ and $\mathrm{CV}<10 \%$, yet with larger variability for mean velocity than for distance covered or propulsive power) and kinetic parameters (ICC $>.94$ and CV $<5 \%$, yet with larger variability for mean horizontal forces than for mean vertical forces) and ranged from good to high for all kinematic $(.88<$ ICC $<.95$ and CV $\leq 3.5 \%)$ and spring-mass variables $(.86<\mathrm{ICC}<.99$ and $\mathrm{CV} \leq 6.5 \%)$. Compared with intrasession, minimal detectable differences were on average twice larger for intersession designs, except for sprint kinetics. Conclusion: Instrumented treadmill sprint offers a reliable method of assessing running mechanics during single sprints either within the same session or between days.
\end{abstract}

Keywords: sprint performance, reproducibility, between sessions, within session, kinetics, kinematics

Sprinting performance is paramount in many sports (eg, track and field, soccer, rugby, Australian Football League, field hockey). This key fitness component is commonly assessed to compare athletes of various standards; ascertain the effects of a given ergogenic supplementation, environmental stress (eg, heat, hypoxia), or training; identify talent; and/or monitor long-term-athlete development (eg, longitudinal studies across age and maturation groups). Assessing sprint performance can be achieved overground through timing lights or, in controlled laboratory test settings, by using torque treadmills ${ }^{1}$ or nonmotorized treadmills. ${ }^{2,3}$ Traditional performance indices include peak velocity, time to peak velocity, distance covered, peak and/or mean power, and different fatigue indexes.

One advantage of treadmill dynamometers that use tethered running over field-based sprinting is the possibility to compute power output as the product of belt velocity and horizontal force (ie, from transducers located at the extremity of the tether). ${ }^{4,5}$ Unless the tether is close to horizontal (during the up-and-down motion of the subject) and short in length, power-output evaluation using such ergometers is flawed; the vertical ground-reaction forces (GRFs) would affect the tether horizontal force as the tethering device is not warranted a horizontal orientation. The measurements of GRF even during off-ground periods and often inaccurate determination of instantaneous values (arbitrary time-window period of $0.25 \mathrm{~s}$ at best) due to rather low sampling frequencies are additional drawbacks of these systems.

An alternative method that overcomes the aforementioned methodological limitations is to conduct testing on a treadmill with built-in

Girard, Brocherie, and Millet are with the Inst of Sport Sciences, University of Lausanne, Switzerland. Morin is with the Laboratory of Human Motricity, Education Sport and Health, University of Nice Sophia Antipolis, Nice, France. Address author correspondence to Olivier Girard at oliv.girard@ gmail.com. force transducers that enable instantaneous 3-directional GRFs and running velocity to be measured continually throughout an entire sprint run. Using an instrumented treadmill that has been modified and validated for sprinting (constant motor torque), ${ }^{3,6}$ our research group has notably documented the running biomechanics during short (100-m), medium (200-m), and long (400-m) sprints ${ }^{7}$ or across series of repeated sprints. ${ }^{8}$ Compared with existing instruments, this treadmill has the advantage of measuring vertical and horizontal forces at the same location and velocity (ie, the contact between the foot and the belt), while instantaneous values are averaged over 1 contact period (ie, not over a constant arbitrary time window).

To ensure confidence in detecting small worthwhile changes in treadmill sprinting performance, the reliability of derived parameters is important but as yet unknown. Although it is widely recognized that power output can be measured reliably in humans tested on such devices, $, 13,9-12$ previous studies mainly investigated the test-retest reliability between days, usually from 2 test sessions separated by about a week. Comparatively, there are currently only limited published data on the reliability of consecutive trials performed the same day. ${ }^{12}$ Nonetheless, a functional-ability screening process is often performed during a single session due to time constraints. Therefore, it is important to know the same-day test-retest (intraday) reliability. In this vein, identification of subgroups is common in a research setting to compare performances between groups at a single time point. Such a diagnostic/discrimination-tool approach would notably ensure identification of subtle differences in key mechanical features within a population of sprinters of various standards. ${ }^{6,13}$ While previous treadmill studies have evaluated power production from running bouts ranging from 20 to 30 seconds, ${ }^{2,14}$ the reproducibility of shorter sprints $(\mathrm{eg},<10 \mathrm{~s}$, representative of team-sport activities) has not been examined.

Arguably, an increased understanding of the mechanical determinants of sprint performance would help scientists and practitioners design more effective training and injury-prevention 
programs ${ }^{4,15}$ In this vein, the inclusion of spring-mass modeling would also be helpful, as it has been used, for instance, to further describe how musculoskeletal stiffness fluctuates during maximal treadmill sprints of various distances. ${ }^{7}$ However, except for the main variables (ie, distance covered, mean and/or peak velocity, or power production), the technological and biological error derived from instrumented treadmills remains to be elucidated, with only the work of Highton et $\mathrm{al}^{9}$ offering a brief view of the reliability for the main spatiotemporal variables (eg, step frequency and step length) over distances of 10, 20, and $30 \mathrm{~m}$.

The aim of the current study was to report the intrasession and intersession reliability of treadmill sprinting-performance indices, associated kinetics and kinematics, and spring-mass variables. If performance and associated sprint mechanics can be measured reliably, as hypothesized, this will pave the way for investigating the effects of various acute and chronic interventions with confidence.

\section{Methods}

\section{Experimental Approach to the Problem}

This study used a repeated-measures design in which the participants, after a period of familiarization, were required to complete 3 testing sessions to determine both intrasession and intersession reliability of performance indices and associated running mechanics of treadmill sprinting. After a standardized warm-up, the first testing session was used to determine intrasession reliability of sprint mechanics from three 5-second sprints interspersed with 2-minute recovery intervals. While single-trial performance assessment may be acceptable after appropriate familiarization and the implementation of standardized warm-up and testing (instructions) protocols, ${ }^{16}$ using a mean score of multiple trials as the basis for estimating sprint performance and its mechanical determinant would reduce measurement error ${ }^{17}$ and is therefore recommended. The average of 3 sprints during 3 separate days, conducted 5 to 7 days apart, was used to assess intersession reliability. This duration is probably optimal for an accurate evaluation of the effects (usually of small magnitude) of an acute intervention (eg, differing environmentally stressing conditions, ergogenic manipulations). Arguably, reliability over shorter $(\leq 3-\mathrm{d})$ or longer $(\geq 10-\mathrm{d})$ periods could be affected to a greater extent by potential residual fatigue or changes in lifestyle, respectively.

\section{Subjects}

Thirteen male recreational team- (football, rugby, basketball) and racket- (tennis, squash) sport players $(31.2 \pm 4.8 \mathrm{y}, 178.4 \pm 6.6$ $\mathrm{cm}, 74.3 \pm 8.2 \mathrm{~kg}, 2-4 \mathrm{~h} / \mathrm{wk}$ of physical activity) participated in the study. They gave their informed, written consent before the commencement of the experiment. The experimental protocol was conducted according to the Declaration of Helsinki for use of human subjects as per the International Journal of Sports Physiology and Performance author guidelines and approved by the Ethics Committee of Shafallah Medical Genetics Center.

\section{Experimental Procedure}

Approximately 48 to 72 hours before data collection, subjects undertook a familiarization session during which they received detailed verbal instructions on the technique required to run on the treadmill. This preliminary visit also enabled the participants to walk and jog at a low intensity before completing short (3- to 5-s) sprints with full recovery at gradually increasing rating of perceived exertion ( 7,8 , and 9 on a modified Borg CR10 scale).${ }^{18}$ Once they became comfortable with the technique required (ie, 7-10 trials were generally required to sprint maximally and to achieve a consistent peak running velocity within a $0.5-\mathrm{m} / \mathrm{s}$ range), the familiarization session was concluded by the completion of 3 maximal sprints separated by 2 minutes of passive rest.

On 3 occasions, separated by 5 to 7 days, participants performed three 5-second treadmill sprints with 2-minute recovery intervals (passive in standing position). Strong verbal encouragement was given during sprint efforts. This was preceded by a warm-up consisting of 10 minutes of running at $10 \mathrm{~km} / \mathrm{h}$, followed by 15 minutes of sprint-specific muscular warm-up exercises (ie, $3 \times$ [high knee, high heels, butt-kick, skipping for $\sim 10 \mathrm{~s}$ with $30 \mathrm{~s}$ walking between], followed by $3 \times$ [3-step accelerations at a subjective sense of effort of 7, 8 , and 9], then by $2 \times$ [3-s sprints at a subjective sense of effort of 8 and 9])..$^{18}$ The participants were instructed to refrain from any strenuous physical activity and maintain their normal diet (avoiding any nutritional supplements or alcohol consumption) during the 2- to 3-week period of testing to avoid any possible interference on their sprinting abilities.

\section{Instrumented Sprint Treadmill}

The sprints were performed on an instrumented motorized treadmill (ADAL3D-WR, Medical Development-HEF Tecmachine, Andrézieux-Bouthéon, France). ${ }^{3,19}$ Briefly, it is mounted on a highly rigid metal frame fixed to the ground through 4 piezoelectric force transducers (KI 9077b, Kistler, Winterthur, Switzerland) and installed on a specially engineered concrete slab to ensure maximal rigidity of the supporting ground. This motorized treadmill allows subjects to sprint and produce realistic acceleration and high running velocities. ${ }^{3,19} \mathrm{~A}$ belt attached to a stiff rope $(1 \mathrm{~cm}$ in diameter, $\sim 2 \mathrm{~m}$ in length) was used to tether subjects to the $0.4-\mathrm{m}$ vertical rail anchored to the wall behind them. When correctly attached, subjects were required to lean forward in a typical and standardized crouched sprint-start position with their left foot forward.

\section{Mechanical Variables}

Mechanical data were sampled at $1000 \mathrm{~Hz}$ continuously over the sprints, and after appropriate filtering (Butterworth-type 30-Hz lowpass filter), instantaneous data of vertical, net horizontal, and total (ie, resultant) GRFs were averaged for each support phase (vertical force above $30 \mathrm{~N})$ over the 5 -second sprints $\left(F_{\mathrm{V}}, F_{\mathrm{H}}\right.$, and $\left.F_{\mathrm{T}}\right)$ and expressed in body weight $(\mathrm{BW})$. The index of force application $\left(D_{\mathrm{RF}}\right)$ representing the decrement in ratio of forces $\left(\mathrm{RF}=F_{\mathrm{H}}: F_{\mathrm{T}}\right)$ with the increasing belt velocity $\left(V_{\text {forward }}, \mathrm{m} / \mathrm{s}\right.$ ) was computed as the slope of the linear RF- $V_{\text {forward }}$ relationship calculated from the step-averaged values between the second step and the step at top $V_{\text {forward }} .{ }^{19}$ These data were collected by measurements of the main step kinematic variables: contact time $\left(t_{\mathrm{c}}, \mathrm{s}\right)$, aerial time $\left(t_{\mathrm{a}}, \mathrm{s}\right)$, swing time $\left(t_{\text {swing }}\right.$, $\mathrm{s})$, step frequency $\left(S_{\mathrm{F}}, \mathrm{Hz}\right)$, and step length $\left(S_{\mathrm{L}}, \mathrm{m}\right)$. Finally, for each 5-second sprint, $F_{\mathrm{H}}$ was used with the corresponding average $V_{\text {forward }}$ to compute net horizontal power $\left(P_{\mathrm{P}}=F_{\mathrm{H}} \times V_{\text {forward }}\right.$, W/kg). Each sprint trial included 15 to 18 ground contacts. After excluding the last 2 ground contacts, the remaining last 3 consecutive steps were used for final analysis of sprint kinetics and kinematics. ${ }^{20}$

A linear spring-mass model of running ${ }^{21}$ was used to investigate the main mechanical integrative parameters characterizing lowerlimb behavior during running. Vertical stiffness $\left(K_{\text {vert }}=F z_{\max }: \Delta z\right.$, $\mathrm{kN} / \mathrm{m})$ was calculated as the ratio of peak vertical forces $\left(F z_{\max }\right.$ in $\left.\mathrm{N}\right)$ 
to the vertical maximal downward displacement of center of mass ( $\Delta z$ in $\mathrm{m}$ ), which was determined by double integration of vertical acceleration of center of mass over time during ground contact. Leg stiffness $\left(K_{\text {leg }}=F z_{\max }: \Delta L\right.$, in $\left.\mathrm{kN} / \mathrm{m}\right)$ was calculated as the ratio of $F z_{\text {max }}$ to the maximum leg spring compression,

$$
\Delta L=\Delta z+L-\sqrt{L_{0}^{2}-\left(\frac{V_{\text {forward }} t_{\mathrm{c}}}{2}\right)^{2}}
$$

in $\mathrm{m}$, both occurring at midstance. Initial leg length $\left(L_{0}\right.$, great trochanter-to-ground distance in a standing position) was determined from subject stature as $L_{0}=0.53 \times$ stature. $^{22}$

\section{Statistical Analysis}

Data used to assess intrasession reliability corresponded to the 3 sprints performed during the first of 3 visits. Intersession reliability was calculated from the average of the 3 sprint trials for each of the 3 test sessions. Intrasession (within-day comparisons) and intersession (between-days comparisons) reliability were analyzed using intraclass correlation coefficient (ICC) for relative reliability and coefficient of variation $(\mathrm{CV}=\mathrm{SD} /$ mean $\times 100)$ for absolute reliability, accordingly to previous test-retest studies on treadmill sprint reliability. ${ }^{14}$ ICCs are more sensitive to systematic bias and can also be used for multiple retests, making them preferred in reliability studies. ${ }^{23,24}$ With $r$ values $>.9, .8$ to .9 , and .7 to .8 for ICCs, reliability was considered high, good, and questionable, respectively. ${ }^{25}$ Concerning $\mathrm{CV}$, a somewhat arbitrary acceptable boundary of $<10 \%$ for reliability has been cited by some authors. ${ }^{23}$ Finally, calculation of the standard error of measurement was used to determine the minimal difference to be confident that a true change in an individual's performance has occurred. ${ }^{26}$ For each variable, minimal detectable difference was calculated by multiplying the standard error of measurement by 1.96 and then by the square root of $2 .{ }^{26}$ Values are expressed as mean \pm SD (CI 95\%). All statistical calculations were performed using SPSS statistical software version 21.0 (IBM Corp, Armonk, NY, USA). The significance level was set at $P<.05$.

\section{Results}

Descriptive data for performance, kinetic, kinematic, and springmass parameters for intrasession and intersession reliability are displayed in Table 1 and Table 2.

All the investigated parameters of the 3 sprint trials performed on the same day exhibited high reliability scores (ICC $>.94$ and $\mathrm{CV}<8 \%$; Table 3). As shown in Table 4, reliability scores for the average values of the 3 sprints performed on different days were good for performance indices $(.83<$ ICC $<.89$ and $\mathrm{CV}<10 \%)$ and kinetic parameters (ICC $>.94$ and $\mathrm{CV}<5 \%$ ) and ranged from good to high for all kinematic variables $(.88<$ ICC $<.95$ and $\mathrm{CV}$ $\leq 3.5 \%)$ and spring-mass characteristics $(.86<\mathrm{ICC}<.99$ and $\mathrm{CV}$ $\leq 6.5 \%$ ) (Table 4). Compared with intrasession (Table 3), minimal detectable differences were on average twice larger for intersession (Table 4) designs, except for sprint kinetics.

\section{Discussion}

To our knowledge, this is the first study to extensively investigate both intrasession (within-day) and intersession (between-days) reliability of performance outcomes and associated running mechanics of treadmill sprinting. Our data indicate that performance, kinetic, kinematic, and spring-mass parameters during treadmill sprinting are highly reliable for both intrasession and intersession designs, exhibiting ICCs of .83 to .99 (above .90 for most variables) and CVs lower than $10 \%$ ( $<5 \%$ for most variables).

There have been several investigations examining the ability of instrumented treadmills to reliably assess performance during tethered sprinting of duration ranging from 3 to 30 seconds. $^{9-12}$ In our relatively homogeneous sample, good to high ICCs were identified for performance outcomes on 3 separate days and over 3 consecutive trials, respectively, with lower ICC and CV for mean velocity than for distance covered or propulsive power. Both Sirotic and Coutts ${ }^{11}$ and Tong et $\mathrm{al}^{12}$ reported that between-days measurements of running velocity $(\mathrm{CV}=1.3 \%$ in those 2 studies) or distance covered were more reliable than for power output $(\mathrm{CV}=5.4 \%$ and $8.2 \%$, respectively) during 6-second treadmill sprints; an opposite trend occurred in our study. Hence, reliability for mean velocity $(\mathrm{CV}=$ $5.1 \%$ ) was lower than for distance covered and propulsive power $(\mathrm{CV}=2.9 \%$ and $2.6 \%$, respectively). However, due to differences in protocol designs (eg, population characteristics, starting positions, sprint durations, and/or treadmill ergometers) direct comparisons between these studies and our results should only remain anecdotal. We note that our data are in line with Morin et $\mathrm{al},{ }^{3}$ who reported an ICC of .90 for propulsive power when examining physical education students over 10-second sprints on an identical treadmill within 2 weeks. Since mean velocity was found less reliable, we recommend that the preferred indices to readily detect small changes in sprint performance on this dynamometer should be the distance covered and the propulsive power.

Developing large horizontal GRFs is crucial to achieve high acceleration during the first few steps (0- to 10 -m distance interval) of a sprint. ${ }^{27}$ While the production of horizontal GRFs progressively decreases throughout the acceleration phase toward maximum running velocity, vertical GRF increases concomitantly to overcome the effects of gravity and thus allow higher running velocity. ${ }^{28}$ Since magnitudes of GRF in both horizontal and vertical directions are ultimately the underlying cause of effective acceleration and maximum velocity sprinting, a careful assessment of their reliability is essential. Unique to the current study was the very high reliability of GRF measurements not only for intrasession but also intersession, yet with mean horizontal GRFs approximately thrice more variable than in the vertical direction. At touchdown, the differences in trunk orientation and lower-limb joint angles, which are largely dependent on ankle-, knee-, and hip-muscle activation levels, may increase the variability in the horizontal distance between the center of mass and toes. ${ }^{29}$

While considering horizontal and vertical GRFs separately is scientifically sound to improve our understanding of sprinting, taking these 2 force components into consideration simultaneously certainly is a more relevant approach. ${ }^{27}$ Hence, the direction of force application seems more important to achieve better sprint acceleration than producing larger impulse, irrespective of its direction, during ground contact. ${ }^{19}$ In this instance, the effectiveness of force application during the acceleration phase, represented here by the $D_{\mathrm{RF}}$ variable, ${ }^{19}$ is paramount for sprint performance. While being the least reproducible performance indices, good (between-days) to high (within-day) reliability scores would still support the use of $D_{\mathrm{RF}}$ to evaluate the effectiveness of force-application technique during sprinting.

Despite increasing interest in musculoskeletal-stiffness regulation during sprinting, ${ }^{7,20}$ no study has investigated the spring-mass characteristics' reliability. While the $\mathrm{CV}$ for the measured variables was remarkably low ( $<2 \%$ for $F z_{\max }$ ), a larger range of $\mathrm{CV}$ values (3.9-6.6\%) was observed for calculated variables of the springmass model, as $K_{\text {vert }}$ and $K_{\text {leg }}$ (especially between days). Recently, these stiffness variables as descriptors of sprint-running mechanics 


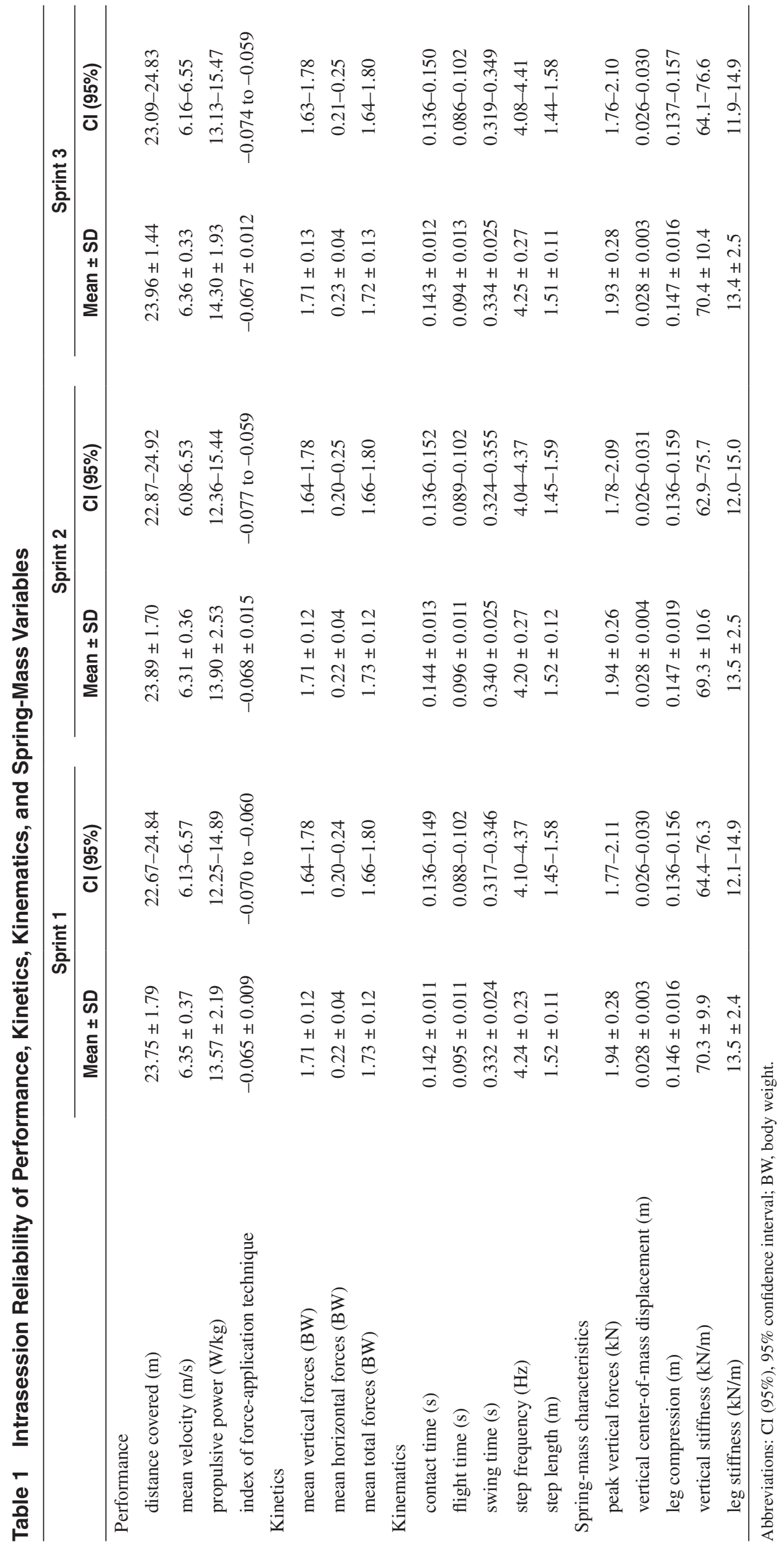




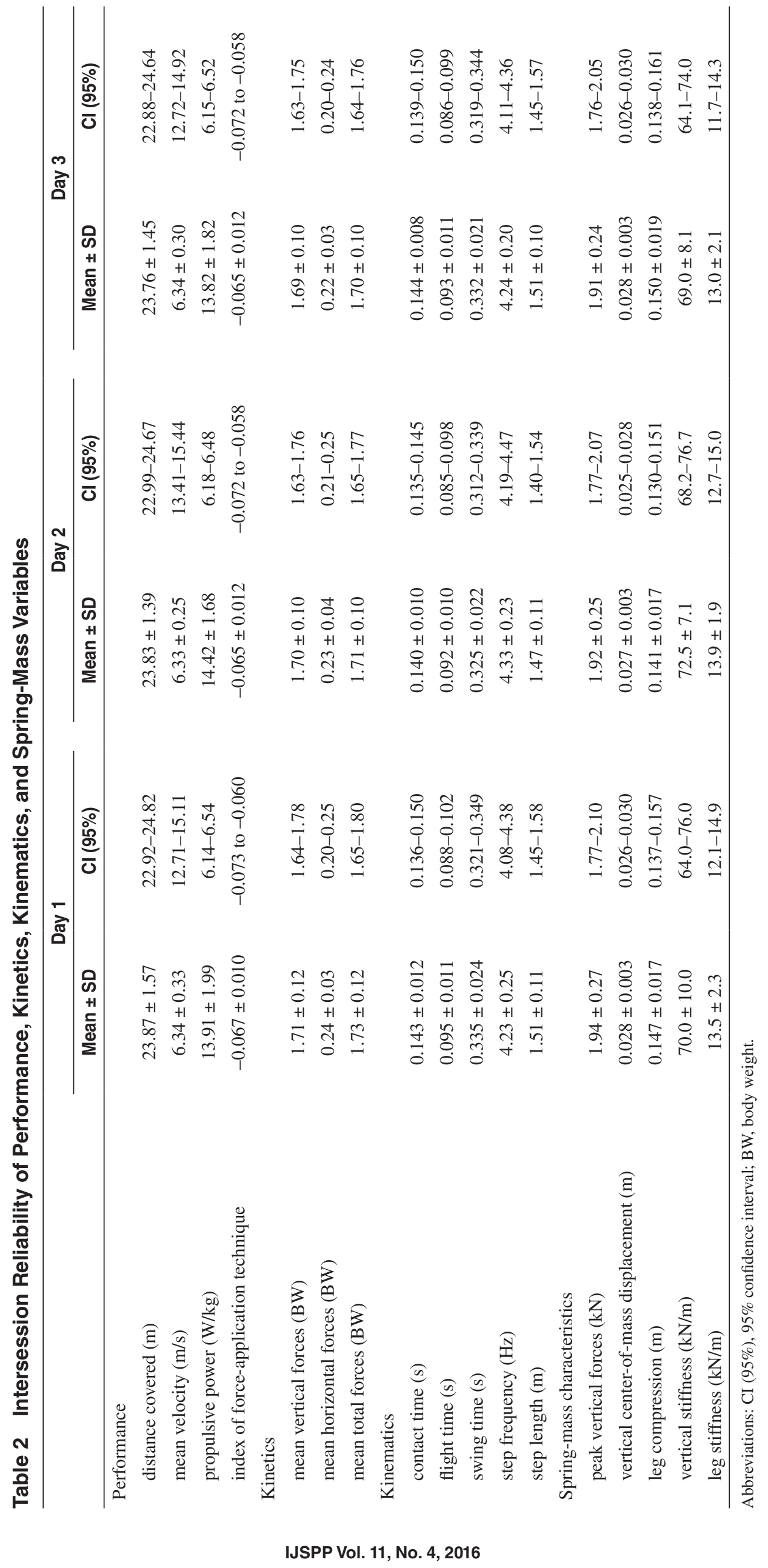


Table 3 Reliability Statistics of the Intrasession Trials for Performance, Kinetics, Kinematics, and Spring-Mass Variables

\begin{tabular}{|c|c|c|c|c|c|c|}
\hline & ICC & $\mathrm{Cl}(95 \%)$ & AIC & CV (\%) & SEM & MDD \\
\hline \multicolumn{7}{|l|}{ Performance } \\
\hline distance covered (m) & .96 & $.90-.99$ & .91 & 2.2 & 0.46 & 1.26 \\
\hline mean velocity $(\mathrm{m} / \mathrm{s})$ & .96 & $.90-.99$ & .89 & 8.0 & 0.09 & 0.24 \\
\hline propulsive power $(\mathrm{W} / \mathrm{kg})$ & .95 & $.86-.98$ & .86 & 2.2 & 0.67 & 1.87 \\
\hline index of force-application technique & .94 & $.86-.98$ & .86 & 8.1 & 0.004 & 0.011 \\
\hline \multicolumn{7}{|l|}{ Kinetics } \\
\hline mean vertical forces (BW) & .98 & $.95-.99$ & .95 & 1.7 & 0.02 & 0.07 \\
\hline mean horizontal forces (BW) & .97 & $.93-.99$ & .92 & 6.8 & 0.01 & 0.03 \\
\hline mean total forces (BW) & .98 & $.94-.99$ & .94 & 1.7 & 0.03 & 0.07 \\
\hline \multicolumn{7}{|l|}{ Kinematics } \\
\hline contact time (s) & .98 & $.95-.99$ & .93 & 2.4 & 0.002 & 0.007 \\
\hline flight time (s) & .98 & $.95-.99$ & .96 & 3.8 & 0.002 & 0.006 \\
\hline swing time (s) & .99 & $.96-1.00$ & .98 & 2.0 & 0.004 & 0.010 \\
\hline step frequency $(\mathrm{Hz})$ & .98 & $.95-.99$ & .95 & 1.8 & 0.05 & 0.14 \\
\hline step length $(\mathrm{m})$ & .98 & $.94-.99$ & .95 & 2.1 & 0.02 & 0.07 \\
\hline \multicolumn{7}{|l|}{ Spring-mass characteristics } \\
\hline peak vertical forces $(\mathrm{kN})$ & 1.00 & $.99-1.00$ & .99 & 1.8 & 26 & 73 \\
\hline vertical center-of-mass displacement (m) & .97 & $.93-.99$ & .92 & 3.6 & 0.001 & 0.002 \\
\hline leg compression (m) & .98 & $.94-.99$ & .94 & 4.3 & 0.004 & 0.011 \\
\hline vertical stiffness $(\mathrm{kN} / \mathrm{m})$ & .98 & $.96-.99$ & .95 & 3.9 & 1.7 & 4.8 \\
\hline leg stiffness $(\mathrm{kN} / \mathrm{m})$ & .98 & $.95-.99$ & .95 & 5.3 & 0.5 & 1.3 \\
\hline
\end{tabular}

Abbreviations: ICC, intraclass correlation coefficient; CI (95\%), 95\% confidence interval; AIC, average intertrial correlation; $\mathrm{CV}$, coefficient of variation; SEM, standard error of measurement; MDD, minimal detectable difference; BW, body weight.

have been questioned by Clark and Weyand, ${ }^{30}$ who compared GRF application across different athletic groups (competitive sprinters vs nonsprint athletes) and running velocity (5 and $7 \mathrm{~m} / \mathrm{s}$ vs individual top velocities). Differences across velocities were observed in competitive sprinters employing an asymmetrical strategy, but not in nonsprinters using patterns of GRFs very close $\left(R^{2}>.90\right)$ to the simple-spring predictions, regardless of whether they were running at top or slower velocities. Although modeling the spring-like behavior of runners is not without limitations ${ }^{21}$ it is likely that, as in our study where team- and racket-sport players executed sprints at mean velocity lower than $6.5 \mathrm{~m} / \mathrm{s}$, this simple spring-mass model remains an acceptable descriptor of stance-limb mechanics.

Overall, our results indicate that both within-day and betweendays reliability are satisfying for most of the tested running mechanical variables, yet with consistently higher scores when sprints are performed on the same day. These findings compare favorably with test-retest data from running at constant $(4.44 \mathrm{~m} / \mathrm{s})$ velocity for 30 seconds $^{31}$ and sprinting for 6 seconds $^{12}$ or over distances of 10, 20, and $30 \mathrm{~m}^{9}$ on nonmotorized treadmills. In addition, the uniqueness of our study was to identify force-production measures to be the most reliable between days when compared with kinematic or spring-mass variables.

The current study has some limitations. First, only 1 familiarization procedure was conducted. While it has also been suggested that performing a familiarization trial before testing for 25 -second tethered running is not necessary to achieve peak performance, ${ }^{14}$ it is commonly recommended that at least 1 but ideally 2 full practice sessions be conducted to best prevent learning (ie, up to $15 \%$ ) or pacing effects, and thereby maximize reliability. ${ }^{10,11}$ Second, the reliability between sessions separated by periods longer than the 5 to 7 days used in the current study remains unknown. Hence, the good to high interday reliability scores would need to be confirmed with testing sessions that are separated by a relatively long duration of time (ie, a few weeks) to accurately evaluate the effects of a training intervention lasting a few weeks. Third, as the current results were obtained in subjects with a team-sport background tested for 5-second sprints, our conclusions should not be extrapolated to other age groups, populations, or sprint durations. Reportedly, the reliability of sprint times (and presumably associated running mechanics) improves with increased distance, ${ }^{9}$ and important differences in mechanical key variables of the sprint have been identified between world-class and subelite sprinters. ${ }^{6,13}$ Finally, given the small sample size often used in sport sciences, future studies would benefit from using confidence intervals, which enable the estimation of random error.

\section{Practical Applications}

Not only are reliability data useful to identify which mechanical parameters are reproducible enough to be considered worthwhile, but they are also important to detect genuine changes in performance. This can be quantified by using the standard error of measurement for defining the minimal difference needed between separate measures to be considered "real" (ie, minimal detectable difference). ${ }^{26}$ When testing on another day, sport scientists should be aware that the acceptability of the measurement error (reliability) 
Table 4 Reliability Statistics of the Intersession Trials for Performance, Kinetics, Kinematics, and Spring-Mass Variables

\begin{tabular}{lcccccc}
\hline & ICC & CI (95\%) & AIC & CV (\%) & SEM & MDD \\
\hline Performance & & & & & & \\
$\quad$ distance covered (m) & .89 & $.71-.96$ & .71 & 2.9 & 0.79 & 2.19 \\
mean velocity (m/s) & .83 & $.55-.94$ & .60 & 5.1 & 0.19 & 0.52 \\
propulsive power (W/kg) & .89 & $.72-.96$ & .74 & 2.6 & 0.95 & 2.65 \\
$\quad$ index of force-application technique & .83 & $.56-.94$ & .60 & 9.7 & 0.007 & 0.020 \\
Kinetics & & & & & & \\
mean vertical forces (BW) & .97 & $.92-.99$ & .92 & 1.4 & 0.03 & 0.09 \\
mean horizontal forces (BW) & .94 & $.86-.98$ & .85 & 4.7 & 0.01 & 0.04 \\
mean total forces (BW) & .97 & $.91-.99$ & .91 & 1.4 & 0.03 & 0.09 \\
Kinematics & & & & & & \\
contact time (s) & .89 & $.71-.96$ & .76 & 3.1 & 0.005 & 0.014 \\
flight time (s) & .95 & $.87-.98$ & .88 & 3.5 & 0.004 & 0.011 \\
swing time (s) & .94 & $.84-.98$ & .89 & 2.2 & 0.008 & 0.022 \\
step frequency (Hz) & .88 & $.70-.96$ & .76 & 2.3 & 0.12 & 0.32 \\
step length (m) & .92 & $.78-.97$ & .82 & 2.9 & 0.05 & 0.13 \\
Spring-mass characteristics & & & & & & \\
peak vertical forces (kN) & .99 & $.97-1.00$ & .97 & 1.7 & 45 & 126 \\
vertical center-of-mass displacement (m) & .86 & $.66-.95$ & .71 & 4.5 & 0.002 & 0.004 \\
leg compression (m) & .91 & $.76-.97$ & .82 & 5.3 & 0.007 & 0.021 \\
vertical stiffness (kN/m) & .91 & $.77-.97$ & .81 & 5.1 & 3.8 & 10.6 \\
leg stiffness (kN/m) & .92 & $.80-.97$ & .83 & 6.5 & 0.9 & 2.5 \\
\hline
\end{tabular}

Abbreviations: ICC, intraclass correlation coefficient; CI (95\%), 95\% confidence interval; AIC, average intertrial correlation; $\mathrm{CV}$, coefficient of variation; SEM, standard error of measurement; MDD, minimal detectable difference; BW, body weight.

of performance indices, spatiotemporal patterns, and spring-masscharacteristic variability decreases (ie, values for standard error of measurement or minimal detectable difference are on average 2 times larger than within the same session), whereas GRFs are highly reliable even on separate days.

Sample-size estimation, using $\mathrm{n}=8 s^{2} / d^{2}$ where $s$ is the typical error (CV\%) and $d$ is the smallest effect, ${ }^{24}$ highlights how $\mathrm{CV}$ likely influences the selection of future dependent variables. Considering the distance covered as the variable of interest, a smallest worthwhile enhancement of $2 \%$ would require 9 participants to detect a "real" difference for sprint tests on the same day, but 17 participants for between-days assessment. The higher variability in mean velocity or spring-mass characteristics (between-days CV $>5 \%$ ) makes it more difficult to observe "real" changes between tests using this arbitrary $2 \%$ threshold. Alternatively, the value of $d$ could be increased for matching sample sizes that are common in most sport-sciences experimental designs. For instance, to detect a $5 \%$ change in mean velocity and $K_{\text {vert }}$ between days, a sample of 8 participants may be sufficient, while 53 and 51 participants would, respectively, be required to detect a $2 \%$ change. Such sample-size estimation reinforces our observation of a higher practicality when expressing the performance alterations by the distance covered or the propulsive power rather than the mean velocity.

Nonetheless, it is known that during tethered sprinting athletes attain only $\sim 70 \%$ to $80 \%$ of their maximum overground velocity. ${ }^{9,10}$ While field- and laboratory-based sprinting performance are equivalent for the majority of key kinematic and kinetic variables, ${ }^{6}$ in the absence of any correction factor, the lower top running velocity attained on the treadmill would lead to inappropriate training recommendations for overground sprinting.

\section{Conclusions}

Reliable running mechanical data can be derived from single sprints on an instrumented treadmill on the same day and between days. Compared with within-day, however, the slightly larger variability associated with between-days designs would imply that larger sample sizes are needed to detect "real" changes in performance or stride parameters due to an intervention.

\section{Acknowledgments}

The authors thank the participants for their enthusiasm and collaboration. This work is based on research funded by QNRF (NPRP 4-760-3-217). The authors have no conflicts of interest nor current or past relationship with companies or manufacturers who could benefit from the results of the current study.

\section{References}

1. McKenna M, Riches PE. A comparison of sprinting kinematics on two types of treadmill and over-ground. Scand J Med Sci Sports. 2007;17(6):649-655. PubMed doi:10.1111/j.1600-0838.2006.00625.x

2. Gonzalez AM, Wells AJ, Hoffman JR, et al. Reliability of the Woodway Curve $^{\mathrm{TM}}$ non-motorized treadmill for assessing anaerobic performance. J Sports Sci Med. 2013;12(1):104-108. PubMed 
3. Morin J-B, Samozino P, Bonnefoy R, et al. Direct measurement of power during one single sprint on treadmill. J Biomech. 2010;43(10):1970 1975. PubMed doi:10.1016/j.jbiomech.2010.03.012

4. de Lacey J, Brughelli ME, McGuigan MR, et al. Strength, speed and power characteristics of elite rugby league players. $J$ Strength Cond Res. 2014;28(8):2372-2375. PubMed doi:10.1519/ JSC.0000000000000397

5. Delextrat A, Baliqi F, Clarke N. Repeated sprint ability and stride kinematics are altered following an official match in national-level basketball players. J Sports Med Phys Fitness. 2013;53(2):112-118. PubMed

6. Rabita G, Dorel S, Slawinski J, et al. Sprint mechanics in world-class athletes: a new insight into the limits of human locomotion. Scand $J$ Med Sci Sports. 2015;25(5):583-594. doi:10.1111/sms.12389

7. Girard $\mathrm{O}$, Brocherie F, Tomazin $\mathrm{K}$, et al. Changes in running mechanics over $100-\mathrm{m}, 200-\mathrm{m}$ and $400-\mathrm{m}$ treadmill sprints [published online ahead of print March 19, 2016]. J Biomech. doi:10.1016/j. jbiomech.2016.03.020

8. Morin J-B, Samozino P, Edouard P, et al. Effect of fatigue on force production and force application technique during repeated sprints. J Biomech. 2011;44(15):2719-2723. PubMed doi:10.1016/j. jbiomech.2011.07.020

9. Highton JM, Lamb KL, Twist C, et al. The reliability and validity of short-distance sprint performance assessed on a nonmotorized treadmill. J Strength Cond Res. 2012;26(2):458-465. PubMed doi:10.1519/ JSC.0b013e318225f384

10. Hopker JG, Coleman DA, Wiles JD, et al. Familiarisation and reliability of sprint test indices during laboratory and field assessment. $J$ Sports Sci Med. 2009;8(4):528-532. PubMed

11. Sirotic AC, Coutts AJ. The reliability of physiological and performance measures during simulated team-sport running on a nonmotorised treadmill. J Sci Med Sport. 2008;11(5):500-509. PubMed doi:10.1016/j.jsams.2007.04.008

12. Tong RJ, Bell W, Ball G, et al. Reliability of power output measurements during repeated treadmill sprinting in rugby players. $J$ Sports Sci. 2001;19(4):289-297. PubMed doi:10.1080/026404101750158394

13. Morin JB, Slawinski J, Dorel S, et al. Acceleration capability in elite sprinters and ground impulse: push more, brake less? J Biomech. 2015;48(12):3149-3154. doi:10.1016/j.jbiomech.2015.07.009. PubMed

14. McLain TA, Wright GA, Camic CL, et al. The development of an anaerobic sprint running test utilizing a nonmotorized treadmill. $J$ Strength Cond Res. 2015;29(8):2197-2204. PubMed doi:10.1519/ JSC.0000000000000854

15. Rumpf MC, Cronin JB, Mohamad IN, et al. The effect of resisted sprint training on maximum sprint kinetics and kinematics in youth. Eur J Sport Sci. 2015;15(5):374-381. PubMed

16. Markwick WJ, Bird SP, Tufano JJ, et al. The intraday reliability of the reactive strength index calculated from a drop jump in professional men's basketball. Int J Sports Physiol Perform. 2015;10(4):482-488. PubMed doi:10.1123/ijspp.2014-0265
17. Mercer TH, Gleeson NP. The efficacy of measurement and evaluation in evidence-based clinical practice. Phys Ther Sport. 2002;3:27-36. doi:10.1054/ptsp.2001.0073

18. Christian RJ, Bishop DJ, Billaut F, et al. The role of sense of effort on self-selected cycling power output. Front Physiol. 2014;5:115. PubMed doi:10.3389/fphys.2014.00115

19. Morin J-B, Edouard P, Samozino P. Technical ability of force application as a determinant factor of sprint performance. Med Sci Sports Exerc. 2011;43(9):1680-1688. PubMed doi:10.1249/ MSS.0b013e318216ea37

20. Brocherie F, Millet GP, Girard O. Neuro-mechanical and metabolic adjustments to the repeated anaerobic sprint test in professional football players. Eur J Appl Physiol. 2015;115(5):891-903. PubMed doi:10.1007/s00421-014-3070-z

21. Coleman DR, Cannavan D, Horne S, et al. Leg stiffness in human running: comparison of estimates derived from previously published models to direct kinematic-kinetic measures. J Biomech. 2012;45(11):1987-1991. PubMed doi:10.1016/j. jbiomech.2012.05.010

22. Winter DA. Biomechanics of Human Movement. 2nd ed. New York: Wiley Science; 1990:75-102.

23. Atkinson G, Nevill AM. Statistical methods for assessing measurement error (reliability) in variables relevant to sports medicine. Sports Med. 1998;26(4):217-238. PubMed doi:10.2165/00007256-199826040-00002

24. Hopkins WG. Measures of reliability in sports medicine and science. Sports Med. 2000;30(1):1-15. PubMed doi:10.2165/00007256-200030010-00001

25. Vincent W. Statistics in Kinesiology. 3rd ed. Champaign, IL: Human Kinetics; 2005.

26. Weir JP. Quantifying test-retest reliability using the intraclass correlation coefficient and the SEM. J Strength Cond Res. 2005;19(1):231240. PubMed

27. Kawamori N, Nosaka K, Newton RU. Relationships between ground reaction impulse and sprint acceleration performance in team sport athletes. J Strength Cond Res. 2013;27(3):568-573. PubMed doi:10.1519/ JSC.0b013e318257805a

28. Weyand PG, Sternlight DB, Bellizzi MJ, et al. Faster top running speeds are achieved with greater ground reaction forces not more rapid leg movements. J Appl Physiol. 2000;89(5):1991-1999. PubMed

29. Kugler F, Janshen L. Body position determines propulsive forces in accelerated running. J Biomech. 2010;43(2):343-348. PubMed doi:10.1016/j.jbiomech.2009.07.041

30. Clark KP, Weyand PG. Are running speeds maximized with simplespring stance mechanics? J Appl Physiol. 2014;117(6):604-615. PubMed doi:10.1152/japplphysiol.00174.2014

31. Pappas P, Paradisis G, Tsolakis C, et al. Reliabilities of leg and vertical stiffness during treadmill running. Sports Biomech. 2014;13(4):391399. PubMed doi:10.1080/14763141.2014.981853 